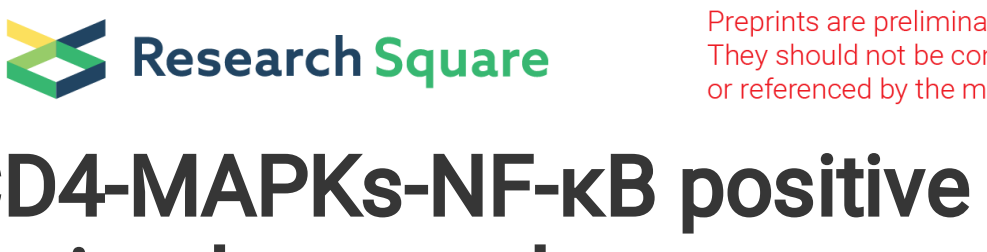 \\ loop simultaneously promotes microglia activation and neuron apoptosis during neuroinflammation
}

\section{Quan Chen}

Hospital 2 of Nantong University, Medical College, Nantong Universityity

Hongjian Lu

Medical Research Center, Affiliated Hospital 2 of Nantong University

\section{Chengwei Duan}

Medical Research Center, Affiliated Hospital 2 of Lanzhou Medical College: Lanzhou University Second

Hospital

\section{Xiangyang Zhu}

Neurology Department, Affiliated Hospital 2 of Nantong University

\section{Yi Zhang}

Neurosurgery Department, Affiliated Hospital 2 of Nantong University

\section{Mengmeng LI}

Medical Research, Affiliated Hospital 2 of Nantong University

Dongmei Zhang ( $\nabla$ zdm_ntyy@163.com )

Nantong City No 1 People's Hospital and Second Affiliated Hospital of Nantong University

https://orcid.org/0000-0002-4132-7363

\section{Research Article}

Keywords: programmed cell death factor 4 (PDCD4), mitogen-activated protein kinases (MAPKs); nuclear factor-kB (NF-kB), microglia, neuroinflammation, neuron apoptosis

Posted Date: March 19th, 2021

DOl: https://doi.org/10.21203/rs.3.rs-304473/v1

License: (1) (i) This work is licensed under a Creative Commons Attribution 4.0 International License.

Read Full License 


\section{Abstract}

Neuroinflammation and neuron injury are common features of the central nervous system (CNS) diseases. It will be of great significance to identify their shared regulatory mechanisms and explore the potential therapeutic targets. Programmed cell death factor 4 (PDCD4), an apoptosis-related molecule, extensively participates in tumorigenesis and inflammatory diseases, but its expression and biological function during CNS neuroinflammation remain unclear. In the present study, utilizing the lipopolysaccharide (LPS)-induced neuroinflammation model on mice, we first reported an elevated expression of PDCD4 in both injured neurons and activated microglia of the inflamed mice brain. A similar change of PDCD4 expression was observed in the in vitro microglial activation model. Silencing PDCD4 by shRNA significantly inhibited the phosphorylation of MAPKs (p38, ERK, and JNK), prevented the phosphorylation and nuclear transportation of NF-kB p65, and thus attenuated the LPS-induced microglial inflammatory activation. Interestingly, LPS also required the MAPKs/NF-kB signaling activation to boost PDCD4 expression in microglia, and thus form a positive loop. Moreover, a persistent elevation of PDCD4 expression was detected in the $\mathrm{H}_{2} \mathrm{O}_{2}$-induced neuronal oxidative damage model. Knocking down PDCD4 significantly inhibited the expression of pro-apoptotic protein BAX, suggesting the proapoptotic activity of PDCD4 in the neuron. Taken together, our data indicated that PDCD4 may serve as a hub regulatory molecule that simultaneously promotes both the microglial inflammatory activation and the oxidative stress-induced neuronal apoptosis within CNS. The microglial PDCD4/MAPKs/NF-KB positive feedback loop may exaggerate the vicious cycle of neuroinflammation and neuronal injury, and thus may become potential therapeutic targets for neuroinflammatory diseases.

\section{Introduction}

Neuroinflammation, an inflammatory reaction occurring within the central nervous system (CNS), extensively participates in CNS pathological processes[1-3]. Microglia, the principal resident immune cells in CNS, accounting for approximately $10 \%$ of brain parenchymal cells, act as the main executor of neuroinflammation[4-6]. Under physiological conditions, microglia secrete multiple neurotrophic factors, dynamically monitor synaptic functions and clear dead cell debris to maintain brain homeostasis. Stimulated by pathogen-associated molecular patterns (PAMPs) following pathogen infection, or by damage-associated molecular patterns (DAMPs) during ischemia, trauma, or neurodegeneration, microglia will activate, proliferate, migrate to the lesion area, and act as the first line of defense in the brain[7,8]. Besides the neuroprotective effects, microglia are the main producer of proinflammatory factors, including interleukin (IL)-1 $\beta$, IL-6, tumor necrosis factor- $a$ (TNF-a), chemokines, reactive oxygen species (ROS), and nitric oxide (NO), which further exacerbate the neuroinflammation and result in the subsequent neuron injury[9-12].

Accumulating evidence suggests that neuroinflammation and neuron injury can form a vicious cycle to promote neuropathological disorders[13]. For example, we previously reported that soluble HSP60 which is released by the injured neurons can act as extracellular DAMPs, combine with the microglial receptor LOX-1, and thus drive neuroinflammation[14][15]. The mitogen-activated protein kinases (MAPKs)family, 
including intracellular signal-regulated kinase 1/2 (ERK1/2), the c-Jun N-terminal (JNK) and p38 MAPK, are major pro-inflammatory signaling pathway within microglia which can be activated by various PAMPs (such as LPS) or DAMPs (such as sHSP60 or the conditioned medium collected from injured neurons)[15] [16]. Following activation, MAPKs subsequently triggers the phosphorylation and nuclear transportation of transcription factors, such as nuclear factor-KB (NF-KB) or activating protein-1 (AP-1), and thereby enhance the transcription of the downstream pro-inflammatory genes[15][16]. Although a large number of studies have focused on the inflammatory activation of microglia, its exact molecular mechanism has not been fully elucidated. Considering the close interaction of neuroinflammation and neuronal injury, it will be of great significance to identify their shared regulatory molecule, analyze their internal relationship, and then explore the potential therapeutic targets.

Human Programmed cell death factor 4 (PDCD4) gene, discovered in 1999, is located on chromosome $3 q 21.3$ with a total length of $28212 \mathrm{bp}$ and encodes a protein containing 496 amino acid residues[17][18]. Based on its pro-apoptotic and cell-cycle inhibiting activity, intensive efforts have been focusing on its role as a tumor suppressor[19][20]. PDCD4 exerts its antineoplastic effects by promoting cellular apoptosis and inhibiting malignant transformation, cellular proliferation, invasion, and metastasis[19][20] [21] Mechanistically, PDCD4 binds to elF4A/E to inhibit the RNA helicase activity or directly interacts with the target mRNAs to block protein translation[18][19]. PDCD4 can also combine with certain cytoplasmic proteins, or interact with some transcription factors and thus modulate the target gene transcription[18] [19].

Besides the tumor-suppressive activity, PDCD4 is also widely expressed in immune cells and closely participates in inflammatory responses, but its role during inflammation is still uncertain. The available conflicting evidence showed the opposite pro-inflammatory or anti-inflammatory effects of PDCD4 under different experimental conditions[18][19]. For example, PDCD4 deficiency protected mice against dietinduced obesity, inflammation in white adipose tissue, and insulin resistance via restoring LXR-a expression[22]. LPS required PDCD4 to induce NF-KB activation and IL-6 expression, and mice deficient in PDCD4 were protected from LPS-induced death[23]. On the contrary, other studies reported that PDCD4 knockout mice displayed up-regulation of pro-inflammatory cytokines (such as IL-6) and enhanced activation of the proinflammatory STAT3activation in the experimental colitis model[24]. The expression and biological function of PDCD4 in CNShas been rarely reported, especially regarding microglial activation and neuroinflammation[25].

In this study, we analyzed the temporal-spatial pattern of PDCD4expression in the classical LPS-induced neuroinflammation model on mice and detected its association with microglial activation and neuronal apoptosis for the first time. Utilizing an in vitro microglial activation model stimulated by LPS, we demonstrated the pro-inflammatory function of PDCD4 in microglia, and also observed its pro-apoptotic activity in the oxidative damaged neuronal model. More importantly, our data suggested that there is a PDCD4-MAPK-NF-KB positive feedback loop to promote the microglial activation and neuronal injury during neuroinflammation. 


\section{Materials And Methods}

\section{Animals and Treatments}

6-8 weeks old male C57BL/6 $(n=63)$ mice were purchased from the Experimental Animal Center of Nantong University (China), and then randomly assigned into 7 groups, including one control group and six experimental groups. The experimental mice were free to access food and water for 7 days in a 12hour light-dark cycle temperature-controlled environment $\left(21^{\circ} \mathrm{C}\right)$. To establish the CNS inflammation model[26], the mice were intraperitoneally injected with $9 \mathrm{mg} / \mathrm{kg}$ of LPS (Escherichia coli 0111-B4, Sigma, St. Louis, MO, USA), and the same amount of normal saline was injected as the control group. The mice were anesthetized at a specific time point after injection to harvest the brain tissue[27]. All the animal experiments were performed. According to the protocol approved by the Institutional Animal Ethics Committee. We tried our best to minimize the number of animals and their suffering in this experiment.

\section{Cell Cultures and Stimulation}

The mouse microglia cell line BV2 and the mouse hippocampal neuron cell line HT22 were cultured with $10 \%(\mathrm{v} / \mathrm{v}$ ) fetal bovine serum and $0.1 \%$ penicillin-streptomycin in DMEM medium (C11995500BT, gibco, China) under an atmosphere of a humidified air and $5 \% \mathrm{CO}_{2}$ at $37^{\circ} \mathrm{C}$. To establish microglial activation model, $100 \mathrm{ng} / \mathrm{ml}$ LPS (L4391, Sigma, USA) was used to stimulate BV2 cells at different time points (Oh, $1 \mathrm{~h}, 3 \mathrm{~h}, 6 \mathrm{~h}, 12 \mathrm{~h}$, or $24 \mathrm{~h}$ ). To mimic the oxidative damage of neurons in vitro, HT22 cells were exposed to various concentrations of $\mathrm{H}_{2} \mathrm{O}_{2}(50,100,200,400,500,100 \mu \mathrm{M})$ for 24 hours, and then select the appropriate concentration of $\mathrm{H}_{2} \mathrm{O}_{2}(400 \mu \mathrm{M})$ to stimulate $\mathrm{HT} 22$ cells at different time points.

\section{Quantitative Real-Time Polymerase Chain Reaction Analysis}

Total RNA from mouse brain tissue and BV2 was extracted using TRIzol (15596018, Ambion, USA) and quantified with a NanoDrop spectrophotometer(NanoDrop ONE C, Thermo Fisher Scientific, USA). Then the cDNA synthesis was conducted using 5x PrimeScript RT Master Mix (TaKaRa) according to the manufacturer's instructions. Quantitative real-time polymerase chain reaction (qRT-PCR) was performed using 2×QuantiNova SYBR Green (QIAGEN) to analyze gene expression on the Bio-Rad CFX Maestro 1.0 system. The relative mRNA expression was analyzed by using the 2- $\triangle \triangle C T$ method and normalized to GAPDH. Primer pair sequences are shown in Table 1.

Table 1. DNA sequences of primers used in PCRs and expected product sizes. 


\begin{tabular}{|llll|}
\hline Forward primer (5'-3') & Reverse primer (5'-3') & \multicolumn{2}{l}{ Size(bp) } \\
GAPDH & CAAGGTCATCCATGACAACTTTG & GtCCACCACCCTGTTGCTGTAG & 496 \\
iNOS & CAAGAGTTTGACCAGAGGACC & TGGAACCACTCGTACTTGGGA & 654 \\
IL-6 $1 \beta$ & GCCTTCTTGGGACTGATGCT & TGGAAATTGGGGTAGGAAGGAC & 475 \\
TNF-a & TCATTGTGGCTGTGGAGAAG & AGGCCACAGGTATTTTGTCG & 333 \\
\cline { 1 - 2 } & ACAGAAAGCATGATCCGCGA & TTGCTACGACGTGGGCTAC & 286 \\
\hline
\end{tabular}

\section{Immunohistochemistry}

Fresh brain tissue was fixed with $4 \%$ paraformaldehyde at $4{ }^{\circ} \mathrm{C}$ overnight and embedded in Tissue-Tek O.C.T compound(SAKURA), then cut into $20 \mu \mathrm{m}$ sections by cryotome sections(CM1860, Leica, Germany). The immunohistochemistry of brain sections was performed according to the DAB Detection Kit's instructions4 (GK600511, Gene Tech(shanghai, China). After returning to room temperature, the sections were washed with phosphate buffer saline(PBS), added endogenous peroxidase blocker and incubated for 20 minutes in the dark, then incubated with anti-PDCD4 $\left(1: 100, C S T\right.$, USA) overnight at $4^{\circ} \mathrm{Cand}$ added with horseradish peroxidase (HRP) at room temperature for 30 minutes, finally, the sections were stained with diaminobenzidine (DAB) and undergo gradient dehydration, drying and resin sealing, The stained sections were observed by microscope(ECLIPSE Ni-U, Nikon, Japan) on NIS-Elements F 4.6 system.

\section{Immunofluorescence Staining}

The brain tissue sections were stained by standard immunohistochemistry procedures. Briefly, the frozen sections were permeabilized in $0.3 \%$ Triton- 100 for 20 min and blocked with $5 \%$ bovine serum albumin (BSA) for $1 \mathrm{~h}$. Next, the sections were incubated overnight at $4{ }^{\circ} \mathrm{C}$ overnight with the following antibodies as appropriate: anti-PDCD4 (Rabbit, 1:200, 9535, CST, USA), anti-PDCD4 (Mouse, 1:100, sc-376430, Santa Cruz, USA), anti-NeuN (Mouse, 1:400, MAB377, Millipore, USA), anti-GFAP (Mouse, 1:400, G3893, Sigma, USA), anti-lba1 (Rabbit, 1:1000, 019-19741, Wako, Japan), anti-iNOS (Rabbit, 1:200, ab178945, Abcam, UK), and anti-Cleaved Caspase-3 (Rabbit, 1:200, 9661s, sigma, USA). Next, the sections were incubated with the proper Alexa secondary antibodies (1:1000, A10042, A21202, A21206, A10037, Thermo Fisher Scientific, USA) at room temperature in the dark for 2 hours, stained the cell nuclei with DAPI (Sigma, USA). All the sections were observed using a fluorescence microscope (ECLIPSE Ni-E, Nikon, Japan) on the NIS-Elements D 5.11 system.

\section{Western Blot}

The lysis buffer (RIPA effective lysis solution: $\mathrm{PMSF}=100: 1)$ was used to lyse and release the proteins in the cells and mouse cerebral cortex tissues at $4{ }^{\circ} \mathrm{C}$, and the supernatant was collected after centrifugation. The protein concentration was determined by the BCA assay $(23225$, ThermoFisher, USA). The proper amount of protein samples were subjected to 10\% SDS-polyacrylamide gel electrophoresis and transferred to polyvinylidene fluoride (PVDF) membrane (Millipore). The membrane was blocked with 
$5 \%$ skim milk for 2 hours, incubated with the specific primary antibody overnight at $4{ }^{\circ} \mathrm{C}$, including antiPDCD4 (9535, CST, USA), anti-iNOS (ab178945, Abcam, UK), anti-IL-1 $\beta$ (ab9722, Abcam, UK), anti-GAPDH (60004-10-Ig, proteintech, China), anti-Cleaved PARP (5625s, CST, USA), anti-phospho-NF-kB p65 (3033, CST, USA), anti-NF-kB p65 (8242, CST, USA), anti-p38 (9212, CST, USA), anti-phospho-p38 (9211, CST, USA), anti-ERK (9102, CST, USA), anti-phospho-ERK (4370, CST, USA), anti-JNK (9252, CST, USA), antiphospho-JNK (92551, CST, USA), anti- $\beta$-actin (60009-1-Ig, proteintech, China), anti-Lamin-B1 (12987-1-AP, proteintech, USA), anti-Bax (2772, CST, USA), followed by the anti-mouse/anti-rabbit secondary antibody for 2 hours. Finally, the protein signal was visualized using an enhanced chemiluminescence system.

\section{Plasmids and Transfection}

PDCD4-shRNA and Neo-shNC (negative control) plasmids were purchased from China Gene Pharma. Use Lipofectamine 3000 Reagent to transiently transfect the plasmids into BV2 cells or HT22 cells according to the manufacturer's instructions.

\section{Table 2. The target sequences of PDCD4-shRNA.}

$$
\begin{aligned}
& \text { sh-PDCD4\#1 5'-GGAAGTGAAGCGGTTAGAAGT-3' } \\
& \text { sh-PDCD4\#2 5'-GGAAGAGGTGGATGTGAAAGA-3' } \\
& \text { sh-PDCD4\#3 5'-GGGACGGTGATGAGCACAAAT-3' } \\
& \text { sh-PDCD4\#4 5'-GCTGCTCTGGATAAGGCTACT -3' }
\end{aligned}
$$

\section{Measurement of Intracellular Reactive Oxygen Species Production}

Intracellular reactive oxygen species (ROS) levels were examined by the DCFH-DA method according to the manufacture's instruction. Briefly, BV2 cells were transfected with the plasmid for 48 hours, stimulated with LPS $(100 \mathrm{ng} / \mathrm{ml})$ for 12 hours, and washed with PBS and treated with serum-free medium containing DCFH-DA (S30033S, Beyotime, China) in the incubator for 20 min, Next, the cells were washed with serum-free cell culture medium for three times and observed using a fluorescence microscope (ECLIPSE Ti-E, Nikon, Japan).

\section{Nuclear and Cytoplasmic Extraction Experiment}

NE-PER Nuclear Protein-Cytoplasmic Protein Extraction Kit (78833, ThermoFish, USA) was used to separate the subcellular fractions according to the manufacturer's instructions. Briefly, BV2 cells were washed with ice-cold PBS and lysed in CER I working reagent protease containing protease inhibitors for 10 min on ice, after adding CER I working reagent, cytosolic protein isolation was performed by centrifuging the supernatant. Then, the addition of the NER working reagent to the cell pellet caused the release of cytoplasmic contents. The protein concentration was quantified using the BCA Protein Assay Kit (23225, ThermoFish, USA). 
MTT analysis was used to evaluate the viability of HT22 cells after exposure to different concentrations of $\mathrm{H}_{2} \mathrm{O}_{2}$. In short, $\mathrm{HT} 22$ cells were seeded in a 96 -well microtiter plate at a density of $0.6 \times 10^{4} \mathrm{celll} / \mathrm{well}$. After treatment with different concentrations of $\mathrm{H}_{2} \mathrm{O}_{2}$, MTT working solution $(5 \mathrm{mg} / \mathrm{ml})$ was added to each welland incubated for 4 hours at $37^{\circ} \mathrm{C}$. Then the plate was added DMSO to dissolve formazan crystals. Finally, the MTT product was calculated with a microplate reader (Synergy H1هBioTek, USA) and analyzed by GraphPad 8.4 system.

\section{Statistical Analysis}

Statistical analysis was conducted using GraphPad Prism 8.4.3 software. The Data were calculated as $($ mean $\pm S D)$. The differences between each group were detected by $t$-tests and $P<0.05$ was considered statistically significant.

\section{Results}

\section{Up-regulated PDCD4 expression in the inflamed mice cerebral cortex following LPS intraperitoneal injection}

To explore the expression and function of PDCD4 in neuroinflammation, we established the classical neuroinflammation model induced by LPS intraperitoneal injection on mice[6]. The mRNA expressions of pro-inflammatory genes (TNF- $\alpha, \mathrm{IL}-1 \beta, \mathrm{IL}-6$, and inducible nitric oxide synthase (iNOS)) in the cerebral cortex of mice were significantly up-regulated by LPS stimulation and reached their peak values around 3d (Fig. 1A). Western blot confirmed the increased levels of pro-inflammatory protein iNOS and proapoptotic protein cleaved-parp in the inflamed cerebral cortex of mice (Fig. 1B). All these results indicated that LPS successfully induced CNS inflammation and neuronal injury in mice. Next, we examined the expression changes of PDCD4 in mice brains by Western blot (Fig. 1B). The PDCD4 level was lower in the cerebral cortex of the saline-injected control group, gradually increased $12 \mathrm{~h}$ after LPS injection, reached the peak at $1 d$, and then decreased back to the basal level by $7 d$. Immunohistochemical staining further clarified the expression and distribution of PDCD4 in the mice brain. Compared with the control group, the number and intensity of PDCD4-positive cells in the cerebral cortex increased significantly $1 \mathrm{~d}$ after LPS administration, which is consistent with the results of Western blot analysis (Fig. 1C).

\section{Elevated PDCD4 expression was associated with neuronal apoptosis and microglial activation in the inflamed mice brain}

To further determine the cell types expressing PDCD4 protein, we employed double immunofluorescence staining and investigated the possible co-localization of PDCD4 with different cell markers, NeuN 
(neuronal marker), GFAP (astrocytic marker), or Iba1 (microglial marker) in mice brain. As shown in Fig. 2, an elevated PDCD4 expression was mainly observed in NeuN-positive neurons and Iba1-positive microglia in the mouse cerebral cortex $1 \mathrm{~d}$ after LPS administration (Fig. 2A, B). No apparent colocalization was detected between PDCD4 and GFAP (the astrocyte marker) (Fig. 2C). Double immunofluorescence staining also demonstrated a clear co-localization of PDCD4 with the inflammatory marker iNOS and cellular apoptotic marker cleaved-caspase3 in the LPS-injected group (Fig. 2D and E). These results indicated that PDCD 4 may be a hub molecule that simultaneously participates in microglial inflammatory activation and neuronal apoptosis in the LPS-triggered neuroinflammation.

\section{Increased PDCD4 expression in the LPS-activated BV2 microglia cells}

To further analyze the contribution of PDCD4 to neuroinflammation, we established an in vitro microglial activation model. LPS $(100 \mathrm{ng} / \mathrm{ml})$ was used to stimulate BV2 microglia cells. Realtime PCR assay demonstrated a time-dependent increase of pro-inflammatory marker genes (iNOS, TNF-a, and IL-1 $\beta$ ) in BV2 cells following LPS administration (Fig. 3A). Western blot analysis confirmed the elevation of the inflammatory proteins (iNOS and IL-1 3 ) with the maximum levels at $12 \mathrm{~h}$ (Fig. 3B). In line with the in vivo results, microglial PDCD 4 expression was significantly induced by LPS stimulation, reached the peak at 12h, and declined thereafter (Fig. 3B). These data further indicated that PDCD4 is closely associated with microglial inflammatory activation.

\section{PDCD4 knockdown suppressed the pro-inflammatory factor expression and ROS production in microglia}

To explore the potential function of PDCD4 in the inflammatory activation of microglia, we silenced PDCD4 expression in BV2 cells by RNA interference (RNAi) and confirmed the interference efficiency by Western blot (Fig. 4A). Since sh-PDCD4\#4 exerted the best silencing effect, it was employed in the next experiments. Western blot revealed that LPS triggered the proinflammatory iNOS expression in BV2 cells (Fig. 4B). RT-PCR also presented the elevated transcription level of pro-inflammatory genes, TNF-a, IL-1 $\beta$, and iNOS (Fig. 4C). Compared with the scrambled control shRNA group (sh-Ctrl), knocking down PDCD4 significantly attenuated the above LPS-induced pro-inflammatory factor expression in BV2 microglia (Fig. 4B and C). Besides, silencing PDCD4 also largely suppressed the LPS-induced ROS production in BV2 cells (Fig. 4D). Thus, although previous reports are conflicting regarding the function of PDCD4 in inflammation, our data demonstrated that PDCD4 facilitates the LPS-induced microglial inflammatory activation.

\section{Silencing PDCD4 inhibited the MAPKs-NF-KB proinflammatory signaling pathway in the LPS-treated microglia}


Next, we investigated the possible downstream signaling pathway through which PDCD4 regulates microglial activation. Western blot analysis showed that LPS sharply induced the phosphorylation of JNK (p-JNK), p38 (p-p38), and ERK (p-ERK) in BV2 cells, indicating the activation of the classical proinflammatory MAPKs signaling pathway (Fig. 5A). Moreover, apparent phosphorylation of NF-KB p65 ( $p$ p65) and its nuclear transportation represented the activation of the pivotal pro-inflammatory transcription factor (Fig. 5A and B). Compared with the control group, silencing PDCD4 significantly inhibited the LPS-induced phosphorylation of all the three MAPKs branches (JNK, p38, and ERK) in BV2 microglia (Fig. 5A). Moreover, knocking down PDCD4 suppressed the NF-KB p65 phosphorylation and its nuclear accumulation in the LPS-treated cells (Fig. 5A and B). Our data suggested that PDCD 4 promoted the LPS-induced microglial activation by up-regulating the classical MAPKs-NF-KB pro-inflammatory signaling pathway.

\section{LPS required the MAPKs-NF-kB signaling pathway to elevate PDCD4 expression in microglia}

To examine the possible mechanism regulating PDCD4 expression in microglia, we utilized the specific inhibitors of MAPKs or NF-KB to pre-treat BV2 cells followed by LPS administration (Fig. 6A). Western blot showed that all the MAPKs inhibitors (SB20358 for p38, PD98059 for ERK, and SP600125 for JNK) prevented the LPS-induced iNOS expression (Fig. 6B), proving their anti-inflammatory function. Interestingly, inhibiting MAPKs signaling pathways significantly reversed the LPS-elevated PDCD4 protein expression in BV2 cells (Fig. 6B). Moreover, specifically inhibiting NF-KB activity by Bay11-7082 also significantly attenuated the LPS-triggered expression of PDCD4 and iNOS (Fig. 6B). Taken together, our results implied that LPS required the MAPKs-NF-KB signaling pathway to elevate PDCD4 expression in microglia, and thus form a positive feedback loop to promote neuroinflammation.

\section{PDCD4 contributes to the oxidative damage of neurons}

It has been well defined that the reactive oxygen species released by activated microglia can greatly damage the neighboring neurons. Since our above in vivo results indicated the co-localization of PDCD4 with NeuN and cleaved-caspase 3 in the inflamed mice brain, we next evaluated the potential role of PDCD4 in the neuronal oxidative damage model in vitro. Different concentrations of hydrogen peroxide $\left(\mathrm{H}_{2} \mathrm{O}_{2}\right)$ were employed to stimulate the mouse hippocampal neuron cell line HT22. MTT assay demonstrated a concentration-dependent decrease of cell viability (Fig. 7A). Since 400 $\mu \mathrm{M} \mathrm{H} 2 \mathrm{O} 2$ treatment for 24 hours resulted in a moderate decline of cell viability (about 40\%) in HT22 cells (Fig. 7A), we chose this condition for the subsequent experiments. Western blot presented a time-dependent induction of the typical pro-apoptotic protein BAX in HT22 cells by H2O2 administration (Fig. 7B). A timedependent continuous elevation of PDCD4 protein was observed in this neuronal oxidative damage model (Fig. 7B). More importantly, knocking down PDCD4 by shRNA significantly inhibited the expression of pro-apoptotic factor BAX in HT22 cells (Fig. 7C). These data indicated that PDCD4 plays a pivotal role 
in promoting reactive oxygen-related neuronal damage, and acts as a shared regulatory molecule for both microglial activation and neuronal injury during neuroinflammation.

\section{Discussion}

Neuroinflammation, the immune response in the nervous system, normally plays a protective role to promote neuronal repair and regeneration. However, persistent neuroinflammation will disturb the brain homeostasis and cause progressive neuron loss and dysfunction, which is a common pathological mechanism for CNS diseases[1][7]. Identifying a shared hub molecule which simultaneously involved in both neuroinflammation and neuronal apoptosis will be of great significance for the in-depth understanding of the pathogenesis of neurological diseases. In this study, we demonstrated that the tumor suppressor PDCD4 acts as a common molecule that simultaneously promotes inflammatory activation of microglia and oxidative stress-mediated apoptosis of neurons. Besides, we proved the existence of a positive feedback loop of PDCD4-MAPK-NF-KB that accelerates the LPS-induced neuroinflammation, which might become a novel therapeutic target for neuroinflammatory diseases.

Programmed cell death 4 (PDCD4), also named MA-3 in mice, was originally cloned as a novel apoptosisinducible gene in the experimental apoptosis models[29]. It is highly conserved during evolution and ubiquitously expressed in normal tissues and organs[8]. Decreased PDCD4 expression was observed in various tumors, such as lung cancer, colorectal cancer, breast cancer, hepatocellular carcinoma, and glioblastoma[19][30][31]. Some pro-apoptotic drugs (such as ionomycin), antineoplastic drugs (such as retinoic acid receptor agonist), or cytokines (including IL-12 and TGF- $\beta 1$ ) can stimulate PDCD4 expression[18][19] Considering its pivotal functions in regulating cellular apoptosis, the potential roles of PDCD4 during neuronal injury has gradually attracted attention. Chronic restraint stress increased PDCD4 expression in mice hippocampus by decreasing the mTORC1-mediated proteasomes degradation[32]. Elevated PDCD 4 expression was observed in the rat spinal cord injury model[33], the chronic sciatic nerve injury (CCI)-induced rat neuropathic pain model[34], the oxygen-glucose deprivation/reoxygenation (OGDR) injury model of rat hippocampal neurons[35], and the ischemia and reperfusion (I/R)-induced neuronal lesion model in mice retinas[25]. The available studies indicate that PDCD4 may be extensively involved in neuronal damage. However, as far as we know, the expression and biological function during LPS-induced neuroinflammation, especially its role in the interaction between neuroinflammation and neuronal injury, have not been systematically studied. In this study, we detected an inducible expression of PDCD4 in the mice neuroinflammation model by LPS intraperitoneal injection, peaked at $1 \mathrm{~d}$, and regressed to the basal line at $7 \mathrm{~d}$. Immunohistochemistry staining revealed its localization in both injured neurons and activated microglia in the inflamed mice brain (Fig. 1 and 2). A similar change of PDCD4 expression was observed in the LPS-induced BV2 microglial inflammatory activation model (Fig. 3). These data indicate a potential involvement of PDCD4 during the LPS-induced neuroinflammation and neuronal injury in mice brain.

Although previous studies implied a close association of PDCD4 with inflammatory diseases, the conflicting experimental data make it difficult to determine its exact biological function and 
mechanisms[8]. Some evidence suggested PDCD4 is a pro-inflammatory molecule. For example, PDCD4 deficiency significantly inhibited the JNK phosphorylation, NF-KB activation, and IL-6 production in mice macrophages[23]. PDCD4 deficient mice were protected from LPS-induced death[20]. PDCD4 overexpression exaggerated the apoptosis and pro-inflammatory cytokine production in toxin-treated intestinal porcine epithelial cells, thereby aggravating cellular damage[36]. However, other results indicated PDCD4 plays an anti-inflammation role. For instance, Pdcd4 deficiency aggravated the experimental colitis and colitis-associated colorectal carcinoma (CRC) in mice via accelerating the typical pro-inflammatory IL-6/STAT3 pathway[24]. In the LPS or D-galactosamine (D-GaIN) induced acute liver injury model, PDCD4-deficient mice presented more necrotic and apoptotic hepatocytes, inflammatory cells infiltration, inflammatory cytokine (IL-6 and TNF-a) release, and liver internal hemorrhage than wild type mice[37]. In the current study, to identify the biological function of PDCD4 in neuroinflammation, we silenced its expression by shRNA and found that knocking down PDCD4 significantly attenuated the expression of pro-inflammatory cytokines (IL-1 $\beta$ and TNFa), iNOS, and ROS production in microglia (Fig. 4). More importantly, silencing PDCD4 inhibited the phosphorylation and activation of all the three substreams of MAPKs signaling (p38, ERK, and JNK), and preventing the phosphorylation and nuclear transportation of NF-KB p65 (Fig. 5). Taken together, our data demonstrated that PDCD4 facilitates the LPS-induced microglial inflammatory activation by promoting the pivotal MAPKs/NF-KB signaling pathway.

The mechanisms that regulating PDCD4 expression during inflammation remain unclear, but its involvement in oncology may provide some clues. PDCD4 expression is reportedly regulated at different levels[18][19]. The epigenetic silence by promoter methylation and some transcription factors such as FOXO and v-myb directly regulate the Pdcd4 expression at the transcriptional level[18][19]. SRSF3 modulates PDCD 4 expression by inhibiting the alternative splicing and nuclear export of Pdcd 4 mRNA at the post-transcriptional level[ $[8,9]$. Some non-coding RNAs, typically miR-21 and IncRNA MALAT1, bind and form sponging to target PDCD4 expression directly at the translational level[18][19]. Moreover, protein-protein interactions (such as BCL6-PDCD4), phosphorylation and ubiquitination modifications reportedly regulate PDCD4 activity, nuclear transfer, and protein degradation at the post-translational level[18][19]. In this study, utilizing the specific chemical inhibitors to pre-treat BV2 cells, we found that specifically blocking the MAPKs ( $\mathrm{p} 38$, ERK, or JNK) or NF-KB activation significantly suppressed the LPSinduced PDCD4 protein expression in BV2 cells (Fig. 6). Our results indicated that LPS requires the MAPKs-NF-KB signaling pathway to stimulate PDCD4 expression, and thus form a PDCD4-MAPKs-NF-KB positive feedback loop to drive the microglial inflammatory activation. Further investigations are needed to explore the exact underlying mechanisms.

PDCD4 was first discovered as an apoptosis-related gene[29], and its involvement in cellular apoptosis has been extensively examined. In Huh7 hepatoma cells, PDCD4 overexpression elevated the expression of the pro-apoptotic member of $\mathrm{Bcl} 2$ protein family $\mathrm{BAX}$, promoted the release of cytochrome $\mathrm{C}$ from mitochondria, and activation of caspases 8,9 , and 3 , and thus accelerated cellular apoptosis[38]. PDCD 4 can specifically bind to the internal ribosome entry site (IRES) elements of both the XIAP and Bcl- $x(L)$ messenger RNAs, represses the translation of these anti-apoptotic proteins by inhibiting the formation of 
the $48 \mathrm{~S}$ translation initiation complex, and thus promotes apoptosis[39]. A recent study implied that miR183-5p may directly target PDCD4 and RIPK3 to protect neurons against apoptosis and necroptosis during amyotrophic lateral sclerosis (ALS)[40]. MiR-21 derived from the exosomes of mesenchymal stem cells (MSCs) suppressed neuronal death via inhibiting PDCD4 and PTEN signaling pathways [17]. Moreover, miR-340-5p performed neuroprotective function by targeting PDCD4 and then activating the $\mathrm{PI3K} /$ Akt pathway in rat hippocampal neurons which exposed to OGDR injury[35]. LncRNA-H19 facilitated PDCD4 expression via sponging miR-21 and regulated the ischemia and reperfusion (I/ $R$ )-induced sterile inflammation and neuronal lesion in mice retinas[25]. Oxidative stress, defined as excess production of reactive oxygen species (ROS), is a typical feature of neuroinflammation, and directly leads to subsequent neuronal damage[4]. In the present study, a persistent elevation of PDCD4 expression was detected in the $\mathrm{H} 2 \mathrm{O} 2$-induced neuron oxidative damage model. Knocking down PDCD4 significantly inhibited the expression of pro-apoptotic protein BAX, suggesting the pro-apoptotic activity of PDCD4 in the oxidative stress-induced neuronal injury (Fig. 7).

In summary, we found that PDCD4 can serve as a hub regulatory molecule that promotes both the microglial inflammatory activation and the oxidative stress-induced neuronal apoptosis within CNS. Moreover, we proved that PDCD4/MAPKs/NF-KB can form a positive feedback loop to exaggerate the vicious cycle of neuroinflammation and neuronal injury, and thus might become the potential therapeutic targets for the treatment of neuroinflammatory diseases.

\section{References}

[1] Xu, Y., M. Z. Jin, Z. Y. Yang, and W. L. Jin. 2021. Microglia in neurodegenerative diseases. Neural Regen Res 16 (2):270-280. doi:10.4103/1673-5374.290881.

[2] Lian, L., Y. Zhang, L. Liu, L. Yang, Y. Cai, J. Zhang, and S. Xu. 2020. Neuroinflammation in Ischemic Stroke: Focus on MicroRNA-mediated Polarization of Microglia. Front Mol Neurosci 13:612439. doi:10.3389/fnmol.2020.612439.

[3] Zhang, W., T. Tian, S. X. Gong, W. Q. Huang, Q. Y. Zhou, A. P. Wang, and Y. Tian. 2021. Microgliaassociated neuroinflammation is a potential therapeutic target for ischemic stroke. Neural Regen Res 16 (1):6-11. doi:10.4103/1673-5374.286954.

[4] Schirmer, Lucas, Dorothy P Schafer, Theresa Bartels, David H Rowitch, and Peter A Calabresi. 2021. Diversity and Function of Glial Cell Types in Multiple Sclerosis. Trends in Immunology.doi:10.1016/j.it.2021.01.005.

[5] Harms, Ashley S, Sara A Ferreira, and Marina Romero-Ramos. 2021. Periphery and brain, innate and adaptive immunity in Parkinson's disease. Acta Neuropathologica:1-19.doi: 10.1007/s00401-021-022685. 
[6] Leng, Fangda, and Paul Edison. 2020. Neuroinflammation and microglial activation in Alzheimer disease: where do we go from here? Nature Reviews Neurology:1-16.doi: 10.1038/s41582-020-00435-y.

[7] Wake, Hiroaki, Hiroshi Horiuchi, Daisuke Kato, Andrew J Moorhouse, and Junichi Nabekura. 2019. Physiological Implications of Microglia-Synapse Interactions. Microglia:69-80.doi: 10.1007/978-1-49399658-2-6.

[8] Chen, Zhihong, and Bruce D Trapp. 2016. Microglia and neuroprotection. Journal of neurochemistry 136:10-17.doi: $10.1111 /$ jnc.13062.

[9] Conti, Pio, Dorina Lauritano, Alessandro Caraffa, Carla Enrica Gallenga, Spiros K Kritas, Gianpaolo Ronconi, and Stefano Martinotti. 2020. Microglia and mast cells generate proinflammatory cytokines in the brain and worsen inflammatory state: suppressor effect of IL-37. European journal of pharmacology 875:173035.doi: 10.1016/j.ejphar.2020.173035.

[10] Barichello, Tatiana, Lutiana R Simoes, Joao Quevedo, and Xiang Y Zhang. 2019. Microglial activation and psychotic disorders: evidence from pre-clinical and clinical studies. Neuroinflammation and Schizophrenia:161-205.doi: 10.1007/7854_2018_81.

[11] Bagheri-Mohammadi, Saeid. 2021. Microglia in Alzheimer's Disease: The Role of Stem Cell-Microglia Interaction in Brain Homeostasis. Neurochemical Research 46 (4):1-8.doi: 10.1007/s11064-020-03162-4.

[12] Simpson, Dominic SA, and Peter L Oliver. 2020. ROS generation in microglia: Understanding oxidative stress and inflammation in neurodegenerative disease. Antioxidants 9 (8):743.doi:

10.3390/antiox9080743.

[13] Hickman, Suzanne, Saef Izzy, Pritha Sen, Liza Morsett, and Joseph El Khoury. 2018. Microglia in neurodegeneration. Nature Neuroscience 21 (10):1359-1369.doi: 10.1038/s41593-018-0242-x

[14] Zhang, D., L. Sun, H. Zhu, L. Wang, W. Wu, J. Xie, and J. Gu. 2012. Microglial LOX-1 reacts with extracellular HSP60 to bridge neuroinflammation and neurotoxicity. Neurochem Int 61 (7):1021-1035. doi:10.1016/j.neuint.2012.07.019.

[15] Ge, X., D. M. Zhang, M. M. Li, Y. Zhang, X. Y. Zhu, Y. Zhou, X. Peng, and A. G. Shen. 2019. Microglial LOX-1/MAPKs/NF-kappaB positive loop promotes the vicious cycle of neuroinflammation and neural injury. Int Immunopharmacol 70:187-200. doi:10.1016/j.intimp.2019.02.013.

[16] Li, M., D. Zhang, X. Ge, X. Zhu, Y. Zhou, Y. Zhang, X. Peng, and A. Shen. 2019. TRAF6-p38/JNK-ATF2 axis promotes microglial inflammatory activation. Exp Cell Res 376 (2):133-148. doi:10.1016/j.yexcr.2019.02.005.

[17] Yoshinaga, H., S. Matsuhashi, C. Fujiyama, and Z. Masaki. 1999. Novel human PDCD4 (H731) gene expressed in proliferative cells is expressed in the small duct epithelial cells of the breast as revealed by an anti-H731 antibody. Pathol Int 49 (12):1067-1077. doi:10.1046/j.1440-1827.1999.00995.x. 
[18] Lu, K., Q. Chen, M. Li, L. He, F. Riaz, T. Zhang, and D. Li. 2020. Programmed cell death factor 4 (PDCD4), a novel therapy target for metabolic diseases besides cancer. Free Radic Biol Med 159:150-163. doi:10.1016/j.freeradbiomed.2020.06.016.

[19] Matsuhashi, S., M. Manirujjaman, H. Hamajima, and I. Ozaki. 2019. Control Mechanisms of the Tumor Suppressor PDCD4: Expression and Functions. Int J Mol Sci 20 (9). doi:10.3390/ijms20092304.

[20] Long, J., Y. Yin, H. Guo, S. Li, Y. Sun, C. Zeng, and W. Zhu. 2019. The mechanisms and clinical significance of PDCD4 in colorectal cancer. Gene 680:59-64. doi:10.1016/j.gene.2018.09.034.

[21] Zhao, Mengxiang, Nisha Zhu, Fengyao Hao, Yuxian Song, and Liang Ding. 2019. The Regulatory Role of Non-coding RNAs on Programmed Cell Death Four in Inflammation and Cancer. Frontiers in Oncology 9:919. doi: 10.3389/fonc.2019.00919.

[22] Wang, Q., Z. Dong, X. Liu, X. Song, Q. Song, Q. Shang, Y. Jiang, C. Guo, and L. Zhang. 2013. Programmed cell death-4 deficiency prevents diet-induced obesity, adipose tissue inflammation, and insulin resistance. Diabetes 62 (12):4132-4143. doi:10.2337/db13-0097.

[23] Sheedy, F. J., E. Palsson-McDermott, E. J. Hennessy, C. Martin, J. J. O'Leary, Q. Ruan, D. S. Johnson, Y. Chen, and L. A. O'Neill. 2010. Negative regulation of TLR4 via targeting of the proinflammatory tumor suppressor PDCD4 by the microRNA miR-21. Nat Immunol 11 (2):141-147. doi:10.1038/ni.1828.

[24] Wang, L., M. Zhao, C. Guo, G. Wang, F. Zhu, J. Wang, X. Wang et al. . 2016. PDCD4 Deficiency Aggravated Colitis and Colitis-associated Colorectal Cancer Via Promoting IL-6/STAT3 Pathway in Mice. Inflamm Bowel Dis 22 (5):1107-1118. doi:10.1097/MIB.0000000000000729.

[25] Wan, P., W. Su, Y. Zhang, Z. Li, C. Deng, J. Li, N. Jiang, S. Huang, E. Long, and Y. Zhuo. 2020. LncRNA $\mathrm{H} 19$ initiates microglial pyroptosis and neuronal death in retinal ischemia/reperfusion injury. Cell Death Differ 27 (1):176-191. doi:10.1038/s41418-019-0351-4.

[26] Salazar, Alexander, Bryan L Gonzalez-Rivera, Laney Redus, Jennifer M Parrott, and Jason C O'Connor. 2012. Indoleamine 2, 3-dioxygenase mediates anhedonia and anxiety-like behaviors caused by peripheral lipopolysaccharide immune challenge. Hormones and behavior 62 (3):202-209.doi: 10.1016/j.yhbeh.2012.03.010.

[27] Zhang, Yu, Xiaojuan Liu, Huaqing Xue, Xiaorong Liu, Aihua Dai, Yan Song, Kaifu Ke, and Maohong Cao. 2016. Upregulation of PRDM5 Is Associated with Astrocyte Proliferation and Neuronal Apoptosis Caused by Lipopolysaccharide. Journal of Molecular Neuroscience 59 (1):146-157.doi: 10.1007/s12031016-0744-5.

[28] Ross, Erika K, Aimee N Winter, Heather M Wilkins, Whitney A Sumner, Nathan Duval, David Patterson, and Daniel A Linseman. 2014. A cystine-rich whey supplement (Immunocal $\left.{ }^{\circledR}\right)$ delays disease onset and 
prevents spinal cord glutathione depletion in the hSOD1G93A mouse model of amyotrophic lateral sclerosis. Antioxidants 3 (4):843-865.doi: 10.1155/2017/3103272.

[29] Shibahara, K., M. Asano, Y. Ishida, T. Aoki, T. Koike, and T. Honjo. 1995. Isolation of a novel mouse gene MA-3 that is induced upon programmed cell death. Gene 166 (2):297-301. doi:10.1016/03781119(95)00607-9.

[30] Long, J., Y. Yin, H. Guo, S. Li, Y. Sun, C. Zeng, and W. Zhu. 2019. The mechanisms and clinical significance of PDCD4 in colorectal cancer. Gene 680:59-64. doi:10.1016/j.gene.2018.09.034.

[31] Li, C., Y. Chen, X. Chen, Q. Wei, R. Ou, X. Gu, B. Cao, and H. Shang. 2020. MicroRNA-183-5p is stressinducible and protects neurons against cell death in amyotrophic lateral sclerosis. J Cell Mol Med 24 (15):8614-8622. doi:10.1111/jcmm.15490.

[32] Li, Y., Y. Jia, D. Wang, X. Zhuang, Y. Li, C. Guo, H. Chu et al. . 2020. Programmed cell death 4 as an endogenous suppressor of BDNF translation is involved in stress-induced depression. Mol Psychiatry. doi:10.1038/s41380-020-0692-x.

[33] Kang, J., Z. Li, Z. Zhi, S. Wang, and G. Xu. 2019. MiR-21 derived from the exosomes of MSCs regulates the death and differentiation of neurons in patients with spinal cord injury. Gene Ther 26 (12):491-503. doi:10.1038/s41434-019-0101-8.

[34] Peng, C., C. Zhang, Z. Su, and D. Lin. 2019. DGCR5 attenuates neuropathic pain through sponging miR-330-3p and regulating PDCD4 in CCl rat models. J Cell Physio/ 234 (5):7292-7300. doi:10.1002/jcp.27487.

[35] Zheng, Yake, Peng Zhao, Yajun Lian, Shuang Li, and Lihao Li. 2019. MiR-340-5p alleviates oxygenglucose deprivation/reoxygenation-induced neuronal injury via PI3K/Akt activation by targeting PDCD4. Neurochemistry International 134:104650.doi: 10.1016/j.neuint.

[36] Gao, X., X. Huang, Q. Yang, S. Zhang, Z. Yan, R. Luo, P. Wang, W. Wang, K. Xie, and S. Gun. 2021. MicroRNA-21-5p targets PDCD4 to modulate apoptosis and inflammatory response to Clostridium perfringens beta2 toxin infection in IPEC-J2 cells. Dev Comp Immunol 114:103849. doi:10.1016/j.dci.2020.103849.

[37] Wang, X., L. Zhang, Z. Wei, X. Zhang, Q. Gao, Y. Ma, X. Liu et al. . 2013. The inhibitory action of PDCD4 in lipopolysaccharide/D-galactosamine-induced acute liver injury. Lab Invest 93 (3):291-302. doi:10.1038/labinvest.2012.174.

[38] Zhang, H., I. Ozaki, T. Mizuta, H. Hamajima, T. Yasutake, Y. Eguchi, H. Ideguchi, K. Yamamoto, and S. Matsuhashi. 2006. Involvement of programmed cell death 4 in transforming growth factor-beta1-induced apoptosis in human hepatocellular carcinoma. Oncogene 25 (45):6101-6112. doi:10.1038/sj.onc.1209634. 
[39] Liwak, U., N. Thakor, L. E. Jordan, R. Roy, S. M. Lewis, O. E. Pardo, M. Seckl, and M. Holcik. 2012. Tumor suppressor PDCD4 represses internal ribosome entry site-mediated translation of antiapoptotic proteins and is regulated by S6 kinase 2. Mol Cell Bio/ 32 (10):1818-1829. doi:10.1128/MCB.06317-11.

[40] Li, C., Y. Chen, X. Chen, Q. Wei, R. Ou, X. Gu, B. Cao, and H. Shang. 2020. MicroRNA-183-5p is stressinducible and protects neurons against cell death in amyotrophic lateral sclerosis. J Cell Mol Med 24 (15):8614-8622. doi:10.1111/jcmm. 15490.

\section{Figures}




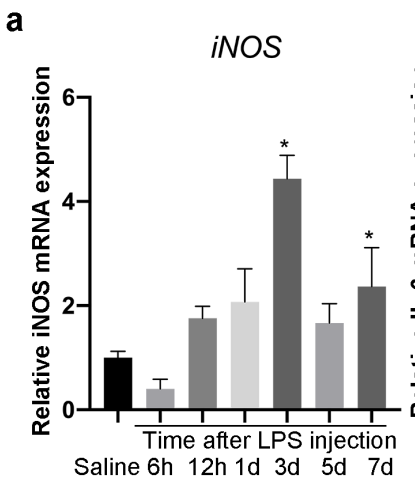

IL-6
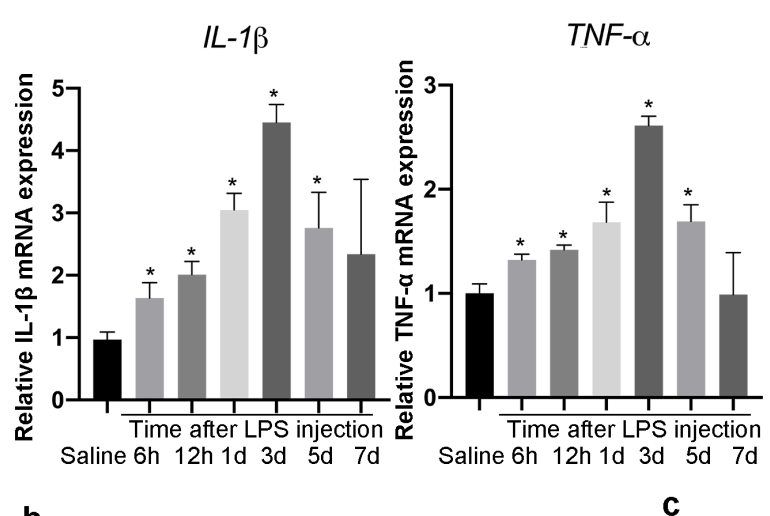

b
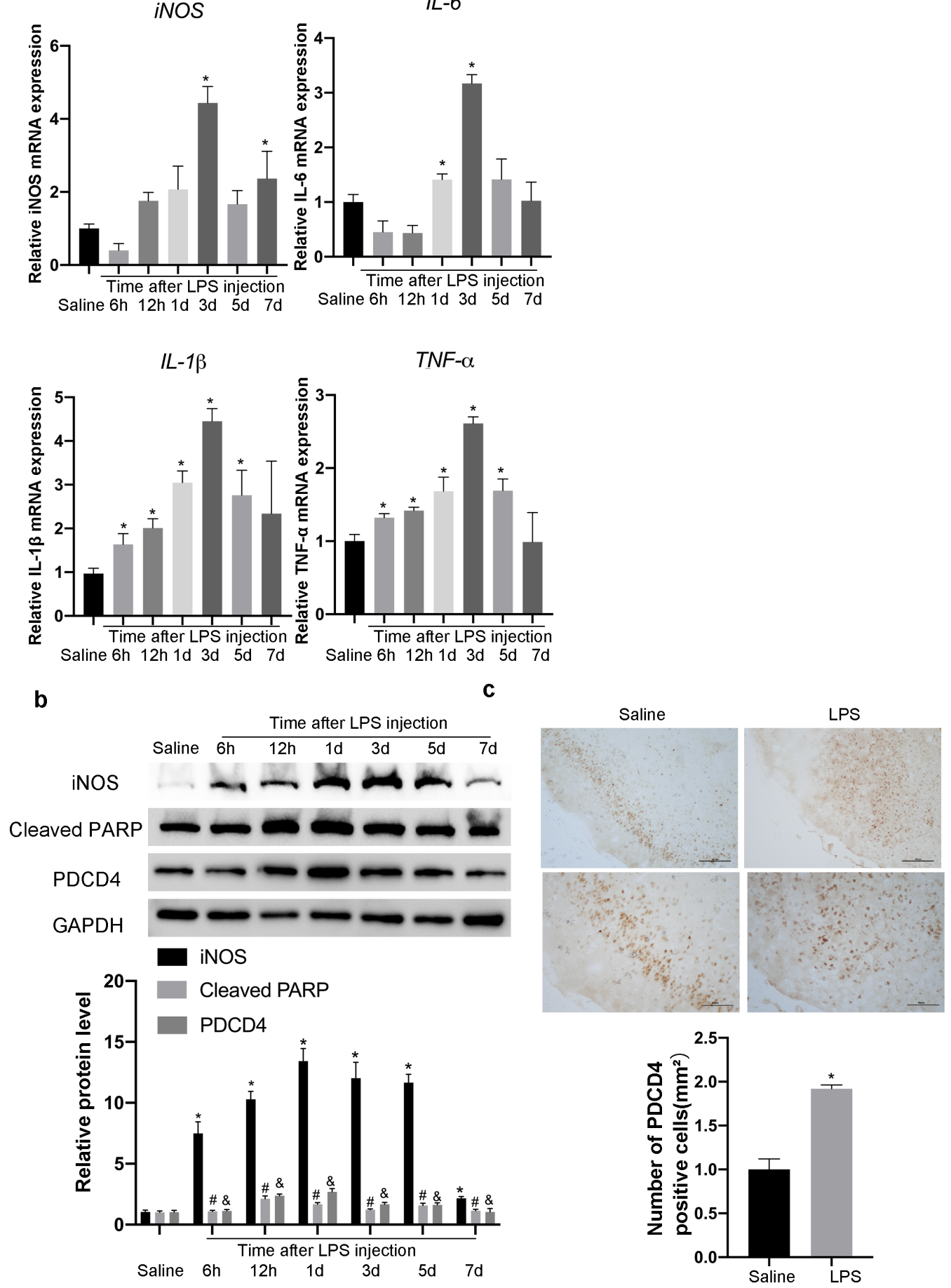

\section{Figure 1}

Elevated PDCD4 expression in the inflamed mice cerebral cortex following LPS intraperitoneal injection. (A) The CNS inflammation model was established by LPS intraperitoneal injection on mice. RT-PCR detected the mRNA levels of TNF-a, IL-1 $\beta$, IL- 6 , and iNOS in the cerebral cortex of mice following LPS treatment. (B) Western blot was used to detect the expression of iNOS, Cleaved-PARP, and PDCD4 in the mice cerebral cortex. (C) Immunohistochemical staining detected PDCD4 expression and distribution in 
either the sham operation group (Saline) or the LPS group 1 day after injection. The value shown is the mean $\pm S E M$ of data from three independent experiments. $\left(n=3,{ }^{\star} p<0.05, \# p<0.05, \& p<0.05\right.$ are significant compared with the control group alone).

a

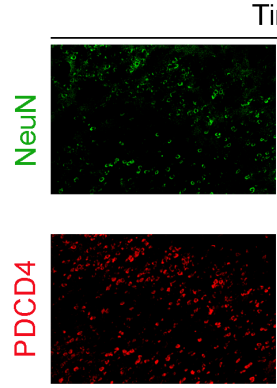

b

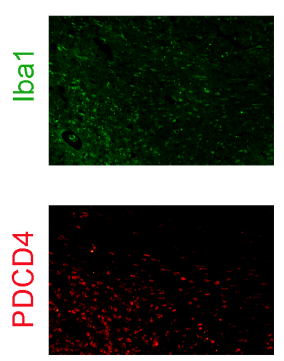

C
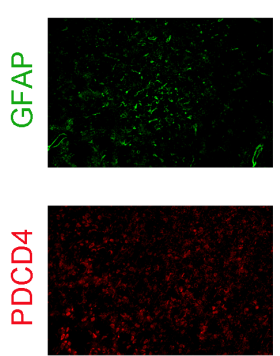

d
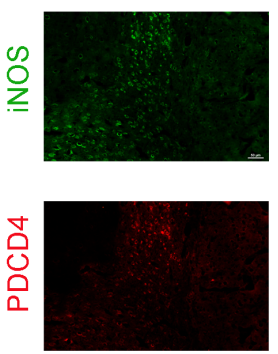

e

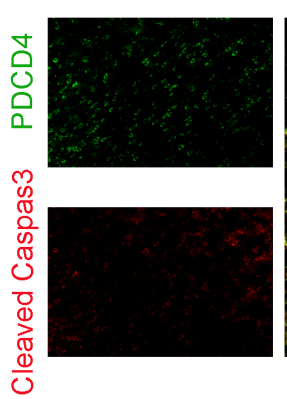

Time after LPS injection
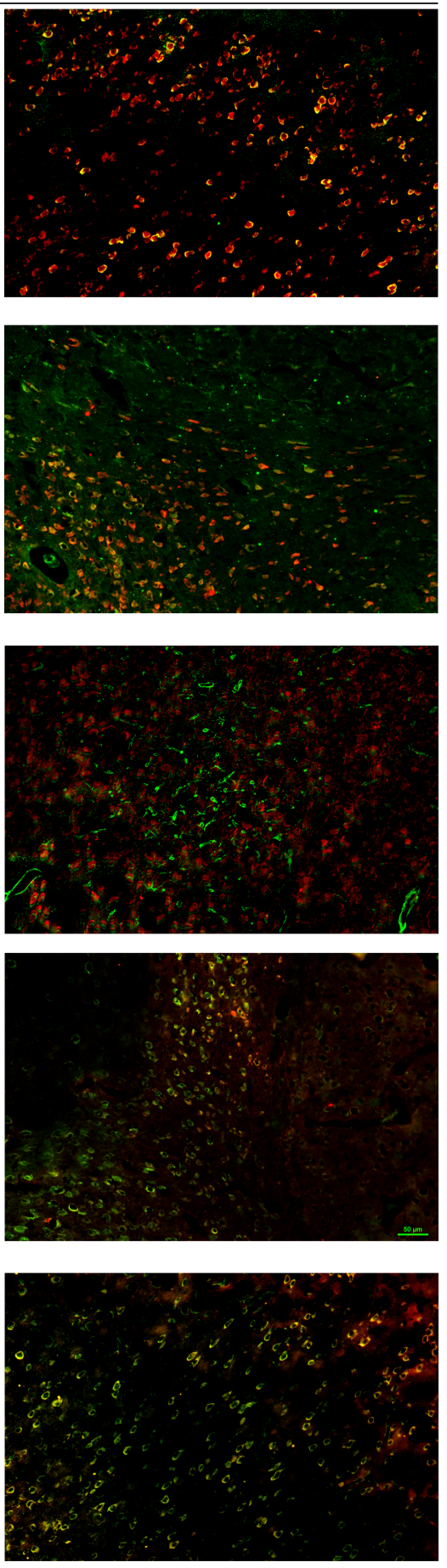

Saline
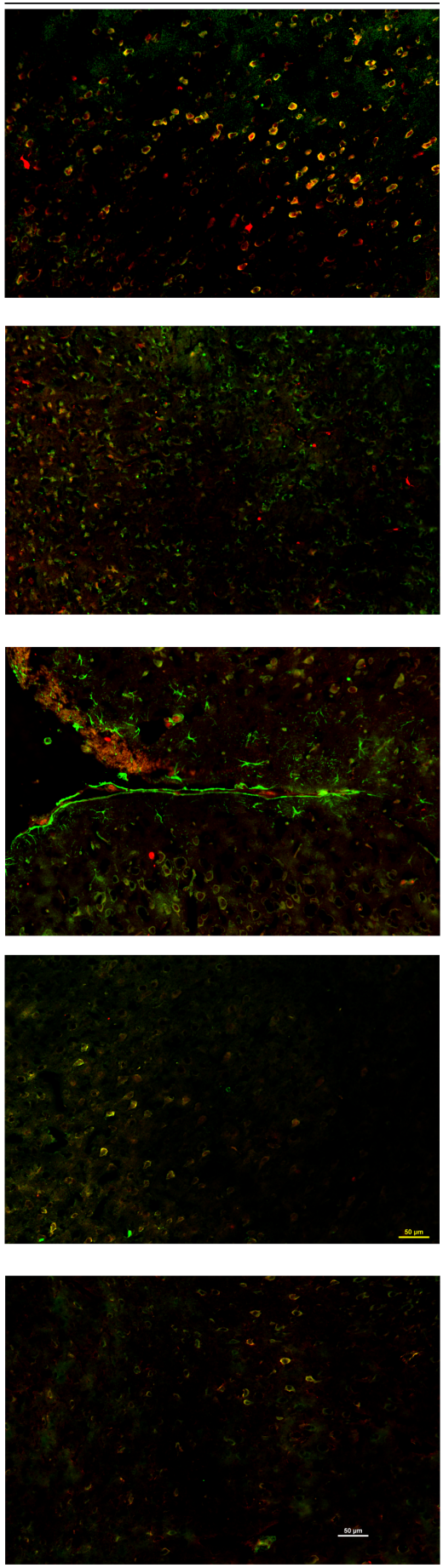

Figure 2

Immunofluorescence staining of PDCD4 and different phenotype-specific markers in the inflamed mouse cerebral cortex. Sections of the mouse cerebral cortex on the first day after LPS injection or the saline 
group were respectively labeled with PDCD4 (red) (green) and different cell markers, such as the neuron marker (A, NeuN, green), the microglia marker (B, Iba1, green), the astrocyte marker (C, GFAP, green), the inflammation-related proteins (D, iNOS, green), or the pro-apoptotic protein (E, Cleaved-Caspase3, red). The scale bar is $50 \mu \mathrm{m}$.

a
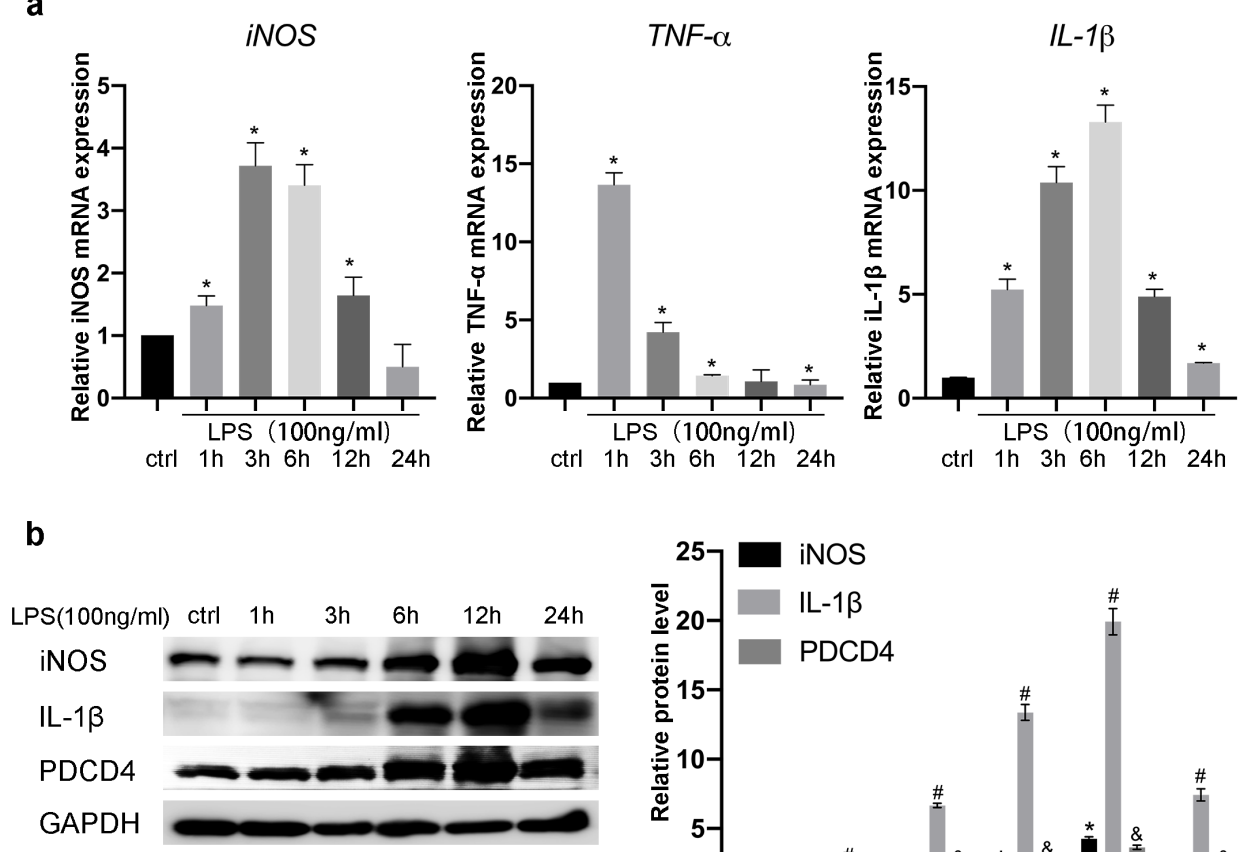

LPS(100ng/ml) ctrl

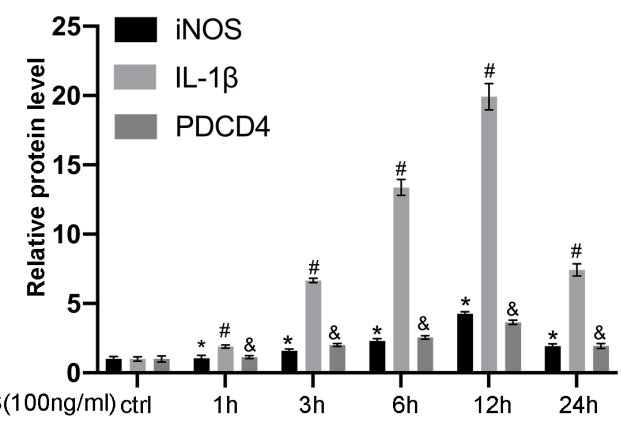

Figure 3 
Up-regulation of PDCD4 in LPS-activated BV2 microglial cells. BV2 microglial cells were treated with LPS (100 ng/ml) for different periods (0h, $1 \mathrm{~h}, 3 \mathrm{~h}, 6 \mathrm{~h}, 12 \mathrm{~h}$, or 24h). (A) RT-PCR detection of iNOS, TNF-a, IL-1 $\beta$ mRNA expression; (B) Western blot analysis of the levels of PDCD4, iNOS, iL-1ßprotein.All data are expressed as the mean $\pm S E M$ of three different experiments. $\left({ }^{\star} p<0.05\right.$, $\# p<0.05$, significantly different from the corresponding control group).

a
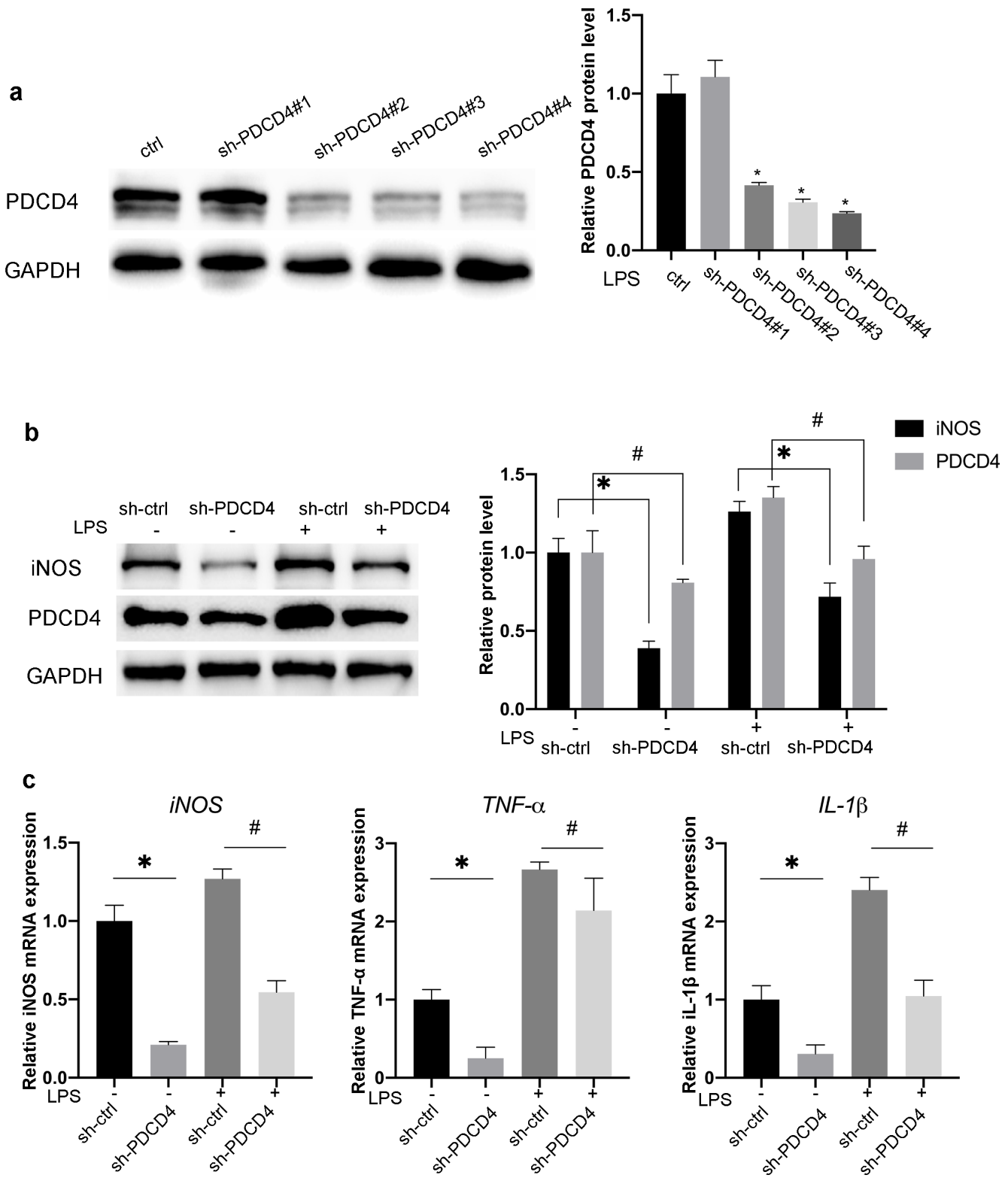

d
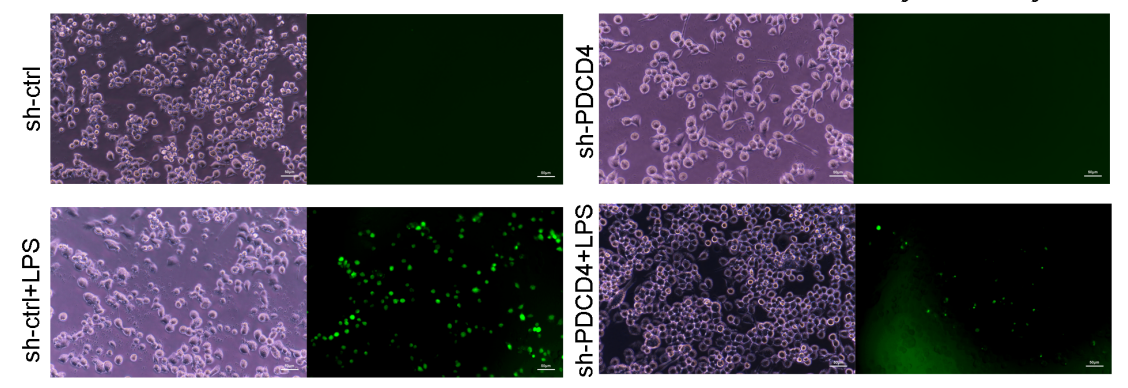

Figure 4 
PDCD4 knockdown reduced the pro-inflammatory cytokine expressionand ROS production in LPSstimulated BV2 microglial cells.(A) The expression of PDCD4 was reduced by transfection with PDCD4shRNA plasmids. Western blot confirmed the knockdown efficiency. Since sh-PDCD4\#4 had the best inhibiting effect on PDCD4 expression, it was used in the following experiments. (B) Western blot showed that knockdown PDCD4 significantly reduced the LPS-induced iNOS expression in BV2 cells. (C) RT-PCR detected the mRNA expression of iNOS, TNF- $\alpha$ and IL-1 $\beta$ in BV2 cells knockdown of PDCD4. (E) DCFH-DA method revealed that knockdown PDCD4 largelyinhibited the production of reactive oxygen species (ROS) inthe LPS-stimulated BV2 cells. The data are expressed as the mean \pm SEM of three different experiments. ( ${ }^{*} p<0.05, \# p<0.05$, there is a significant difference from the corresponding control group). 


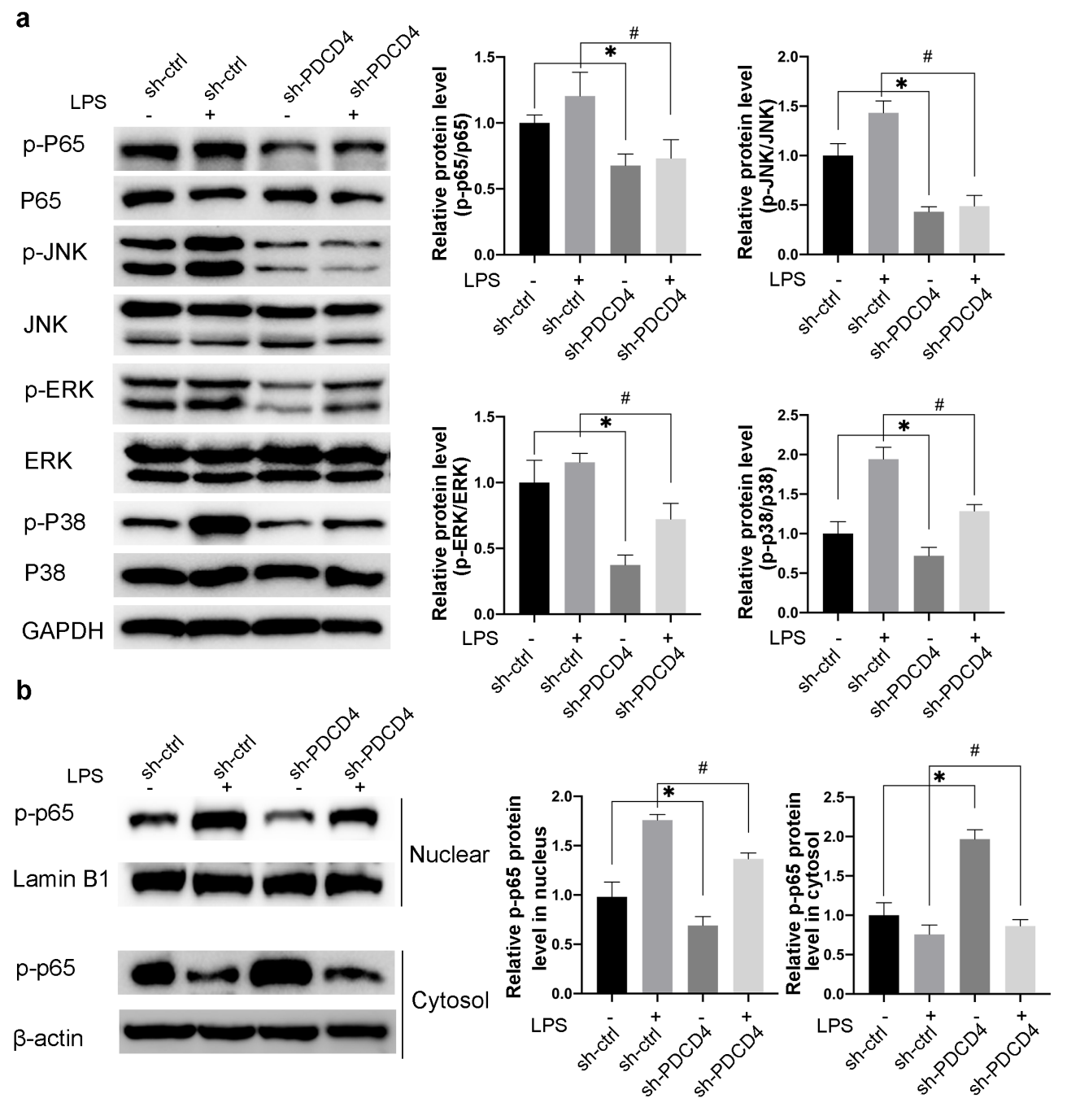

\section{Figure 5}

Silenced PDCD4 significantly inhibited the MAPK/NF-KB signaling activation in LPS-stimulated BV2 cells. (A) BV2 microglial cells were stimulated by LPS for 3 hours. Western blot showed that inhibiting PDCD4 expression by shRNA significantly reduced thephosphorylation ofNF-kB p65 (p-p65) and the phosphorylation of MAPKs ( $p-J N K, p-E R K, p-P 38$ ) in BV2 cells either with or without LPS treatment. (B) The protein levels of NF-kBp65 and p-p65 in the cytoplasm and nucleus of BV2 were also detected by 
Western blot. The data are expressed as the mean \pm SEM of three different experiments. $\left({ }^{*} p<0.05, \# p<0.05\right.$, there is a significant difference from the corresponding control group).

a
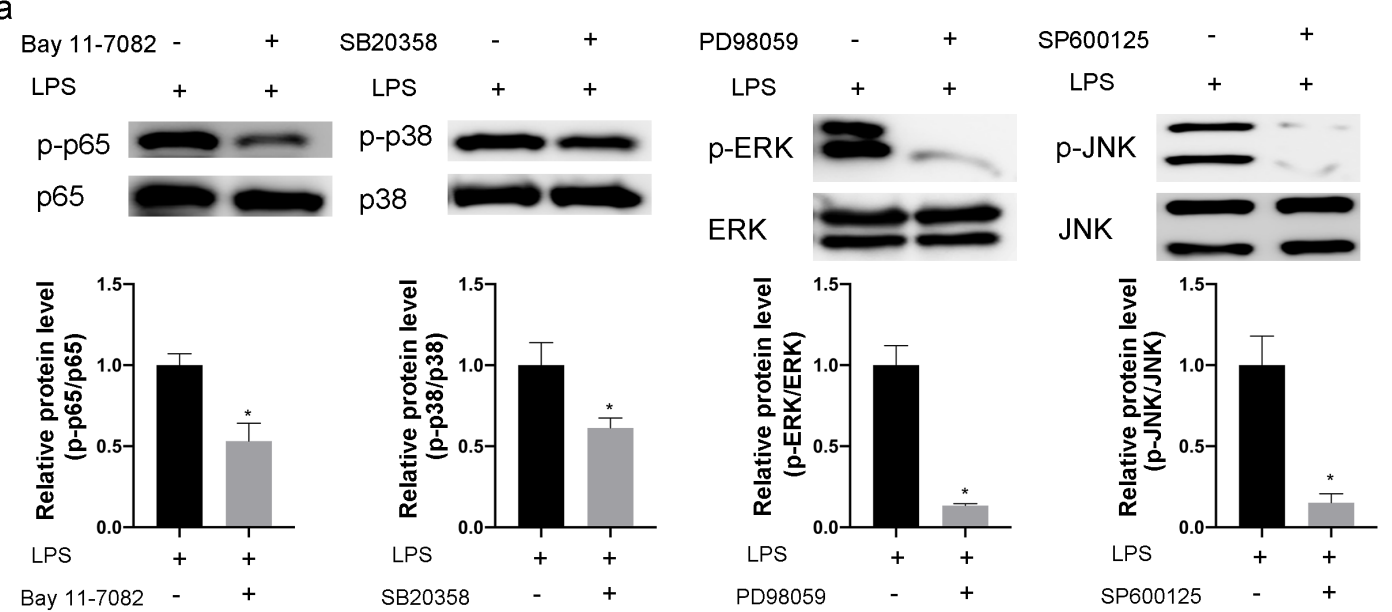

b
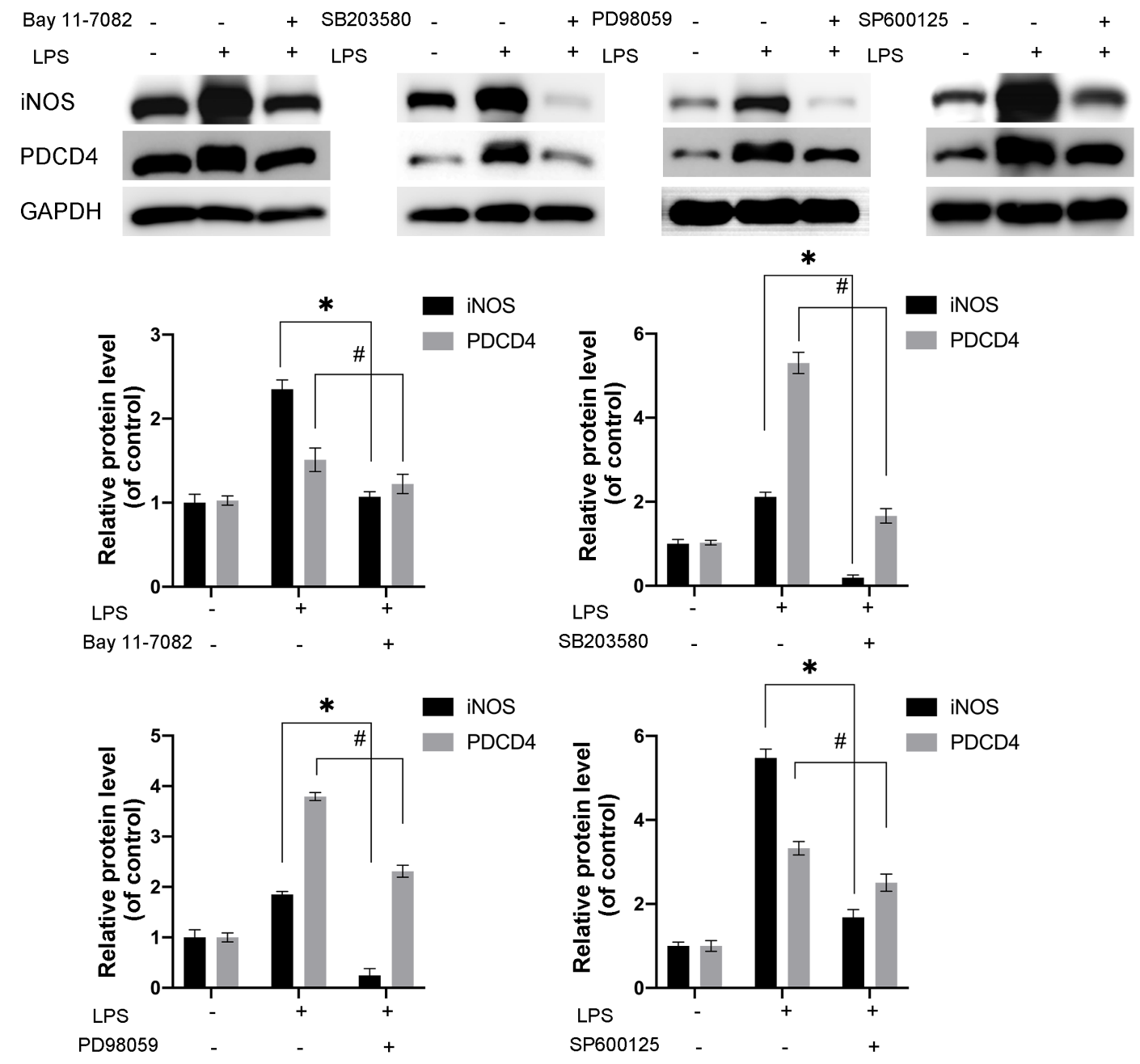

Figure 6

The expression of PDCD4 in microglia was positively regulated by MAPK /NF-KB signaling. (A) BV2 cells

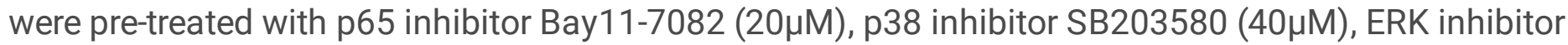
PD98059, or JNK inhibitor SP600125 for 1h, followed by LPS-stimulation for 3h. Western blot analysis 
confirmed the inhibiting effect on the phosphorylation of p65, p38, ERK or JNK. (B) The protein expression of iNOS and PDCD4 were analyzed by Western blot. The LPS-stimulated PDCD4 expression in BV2 cells was largely reduced by the above inhibitors. The data are expressed as the mean \pm SEM of three different experiments. $\left({ }^{*} p<0.05, \# p<0.05\right.$, there is a significant difference from the corresponding control group).
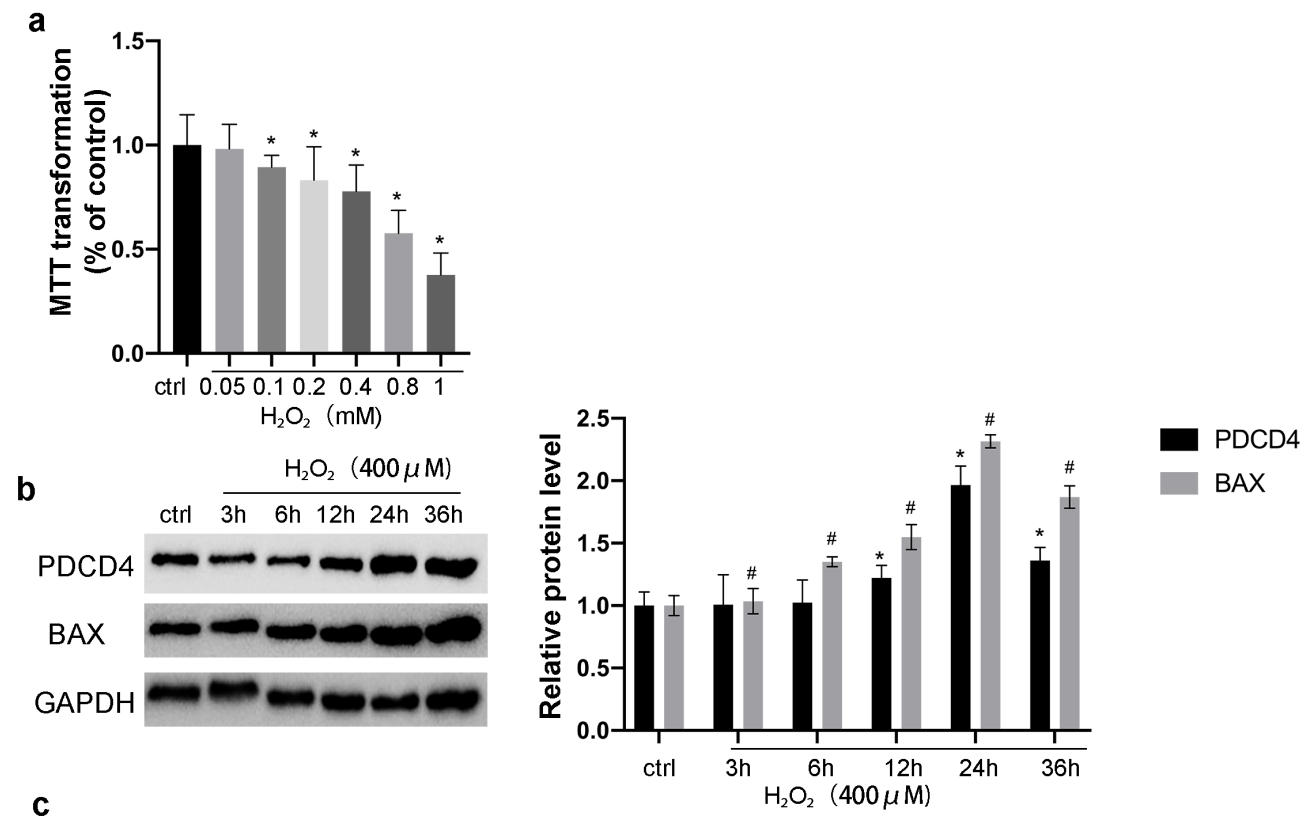

C

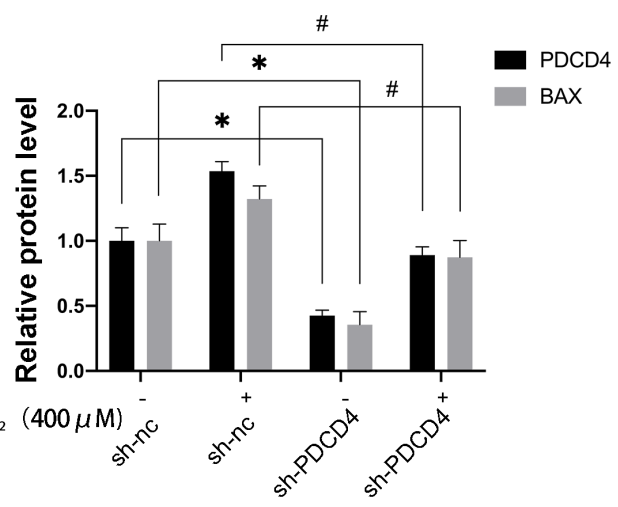

Figure 7 
Silencing PDCD4 attenuatedthe hydrogen peroxide (H2O2)-induced apoptosis of HT22 neuron cells.(A) The mouse hippocampal neuron cell line HT22 was treated with different concentrations of $\mathrm{H} 2 \mathrm{O} 2(50 \mu \mathrm{M}$, $100 \mu \mathrm{M}, 200 \mu \mathrm{M}, 400 \mu \mathrm{M}, 800 \mu \mathrm{M}, 1000 \mu \mathrm{M})$ for $24 \mathrm{~h}$. MTT assaydetected the concentration-dependent

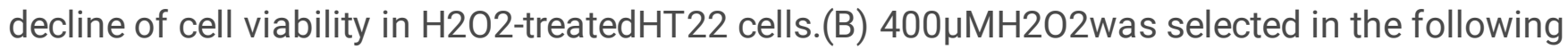
experiments to establish the neuronal apoptotic experimental model. Western blot analysis demonstrated a time-dependent increase of both PDCD4 and the pro-apoptotic protein Bax. (C) Silencing PDCD4 byshRNA significantly inhibited the H2O2-induced BAX expressionin HT22 cells. The data are expressed as the mean $\pm S E M$ of three different experiments. $\left({ }^{*} p<0.05, \# p<0.05\right.$, there is a significant difference from the corresponding control group). 